\title{
PER TONE SHAPING FILTERS FOR DMT TRANSMITTERS
}

\author{
Chun-Yang Chen, See-May Phoong
}

\author{
Dept. of EE \& Graduate Inst. of Comm. Engr. \\ National Taiwan Univ. \\ Taipei, Taiwan, ROC 106
}

\begin{abstract}
Discrete MultiTone (DMT) modulation has been widely adopted for wideband transmission over digital subscriber loop (DSL) channels. In the conventional DMT system, the transmitting filters are the DFT filters. DFT filters suffer from poor frequency responses. Hence the resulting transmit signal has a large spectral sidelobe and its power spectral density (PSD) decays at a rate of $1 / f$ only. In this paper, we propose a modified DMT scheme with improved transmitting filters. In the proposed system, shaping filters are applied to improve the frequency responses of the transmitting filters. Moreover, an efficient implementation of the proposed system is derived. Experiment shows that for VDSL applications, the proposed system has a very satisfactory spectral efficiency.
\end{abstract}

\section{INTRODUCTION}

The DMT or OFDM (Orthogonal Frequency Division Multiplexing) system has enjoyed great success in wideband transmission over wired and wireless communications [1]. Fig. 1(a) shows the block diagram for a DMT transceiver. At the transmitter, the input vector containing $M$ data symbols (QAM or PAM) is first processed by an $M$-point IDFT operation. The output vector of the IDFT matrix is converted to a serial sequence and a cyclic prefix (CP) of length $L$ is added before transmission. At the receiver, the first $L$ samples corresponding to the $\mathrm{CP}$ in each received block are removed and the remaining $M$ samples are processed by an $M$ point DFT operation. It is well-known that when the order of the channel $^{1}$, which is often assumed to be an LTI system, is $\leq L$, we can obtain a zero-forcing (or ISI-free) receiver by multiplying the DFT output by a set of scalars $\lambda_{k}$ known as the frequency domain equalizer [1], as shown in Fig. 1(a).

Using multirate identities [2], one can show that the DMT system shown in Fig. 1(a) is a special type of filter bank transceiver. The transmitter of the DMT system can be redrawn as Fig. 1(b), where the interpolation ratio $N=M+L$. The filters $F_{k}(z)$ are the transmitting filters and they are the DFT filters:

$$
F_{k}(z)=\alpha^{k L} \sum_{l=0}^{N-1} \alpha^{-l k} z^{-l}=\alpha^{k L} F_{0}\left(\alpha^{k} z\right)
$$

This work was supported in parts by National Science Council, Taiwan, ROC, under NSC 92-2219-E-002-015, Ministry of Education, Taiwan, ROC, under Grant 89E-FA06-2-4.

${ }^{1}$ For DSL applications where the channels have long impulse responses, time domain equalizers are often used to shorten the channel responses[1]. where $\alpha=e^{-j 2 \pi / M}$. The DFT filters have poor frequency responses; the first sidelobe is only $-13 \mathrm{~dB}$ and the stopband decays at a rate of $1 / f$ only. In many applications, the PSD of the transmitting signal is not allowed to exceed a predefined spectral mask. To satisfy the PSD constraint, many tones (or subchannels) cannot be used for data transmission and this results in a significant loss of bandwidth. Several methods have been proposed to improve the transmitting filter responses. In particular, the time-domain windowing method (see Sec. II), which has been adopted in the VDSL standard [3], is attractive due to its low complexity. However in this case the shaping filters are restricted to frequency shifted versions of a single prototype filter; we are not able to shape each individual transmitting filter according to the spectral mask requirement.

In this paper, we propose a per tone shaping filter technique to improve the transmitting filter responses. The proposed DMT system with per tone shaping filters includes the time-domain windowing method as a useful special case. In the proposed method, each transmitting filter is shaped by a different filter. Each shaping filter can be optimized separately so that the PSD satisfies the given spectral mask. To reduce the computational load, an efficient implementation is derived for the proposed transmitter. Compared with the conventional DMT scheme, the additional complexity is only $M$ times the shaping filter length for every input vector. As we will see, the use of shaping filters will not change SNR of each tone. Numerical experiment is carried out for the spectral mask requirement in VDSL standard [3]. The results show that the proposed method has a significant improvement over the time-domain windowing method.

\section{PER TONE SHAPING FILTERS}

Fig. 2 shows the block diagram for the per tone shaping filter scheme. There are a total of $M$ tones and the upsampling ratio is $N$. The number of redundant samples is therefore $N-M$. In the proposed transmitter, each tone can have a different shaping filter

$$
W_{k}(z)=\sum_{n=0}^{P} w_{k}(n) z^{-n} .
$$

For convenience, we assume that all the windows have the same order $P$. The case of unequal filter length can be incorporated by simply setting some coefficients to zero. The $k$ th transmitting filter becomes

$$
\widetilde{F}_{k}(z)=F_{k}(z) W_{k}(z) .
$$

Let the channel be an LTI channel $C(z)$. Then the equivalent channel seen by the $k$ th tone is $C(z) W_{k}(z)$. If the order of $C(z)$ is 


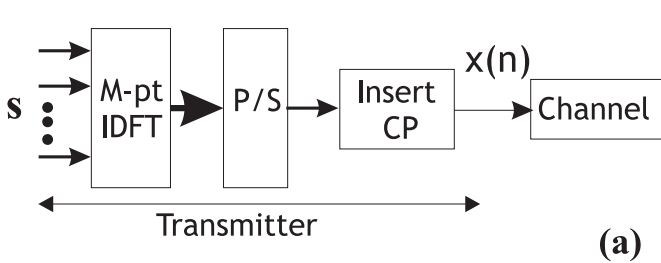

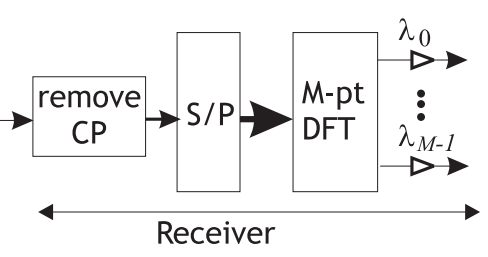

(a)

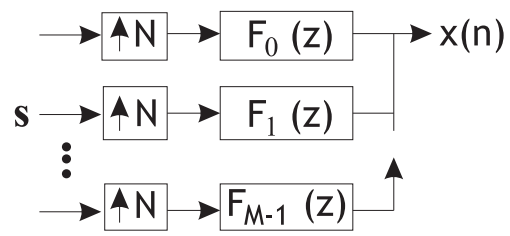

(b)

Fig. 1. (a) DMT system (b) Filterbank representation of the transmitter.

$\leq L$, where

$$
L=N-M-P .
$$

Then one can verify that if we set the FEQs in Fig. 1(a) to

$$
\lambda_{k}=\frac{1}{C\left(e^{j 2 \pi k / M}\right) W_{k}\left(e^{j 2 \pi k / M}\right)},
$$

then the receiver in Fig. 1(a) is a zero forcing receiver for the transmitter in Fig. 2. If the shaping filters satisfy

$$
W_{k}\left(e^{j 2 \pi k / M}\right)=1, \text { for all } k,
$$

then then the FEQs become $\lambda_{k}=1 / C\left(e^{j 2 \pi k / M}\right)$. Hence the noise gain for the DMT system with per tone windows is identical to that of the conventional DMT system.

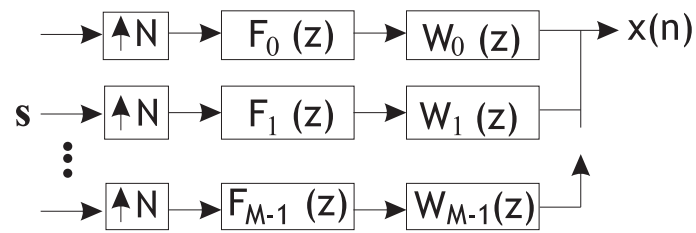

Fig. 2. DMT transmitter with pertone window $W_{k}(z)$

Time-Domain Windowing Method: In VDSL standard [3], the time-domain windowing method is adopted to improve the response of transmitting filters. In this case, the shaping filters are frequency shifted versions of a single prototype filter. To be more specific, they are related by ${ }^{2}$

$$
W_{k}(z)=\alpha^{-k L} W_{0}\left(\alpha^{k} z\right),
$$

where $\alpha=e^{-j 2 \pi / M}$. The main advantage of frequency shifted filters is its low implementational complexity. Using (1) and (4), the transmitting filters are also frequency shifted versions of a single prototype filter: $\widetilde{F}_{k}(z)=\widetilde{F}_{0}\left(z \alpha^{k}\right)$. Let us decompose $\widetilde{F}_{0}(z)$ into its polyphase components as $\widetilde{F}_{0}(z)=\sum_{i=0}^{M-1} E_{0}\left(z^{M}\right) z^{-i}$. Then we have

$$
\left(\begin{array}{c}
\widetilde{F}_{0}(z) \\
\widetilde{F}_{1}(z) \\
\vdots \\
\widetilde{F}_{M-1}(z)
\end{array}\right)^{T}=\left(\begin{array}{c}
E_{0}\left(z^{M}\right) \\
z^{-1} E_{1}\left(z^{M}\right) \\
\vdots \\
z^{-M+1} E_{M-1}\left(z^{M}\right)
\end{array}\right)^{T} \boldsymbol{\Phi}^{H}
$$

${ }^{2}$ We have included a scaling factor $\alpha^{-k L}$ for notational simplicity. This will not affect the system performance. where $\boldsymbol{\Phi}$ is the $M$ by $M$ DFT matrix whose $m n$th entry is given by $[\boldsymbol{\Phi}]_{m n}=\alpha^{m n} / \sqrt{M}$. Recall that $\widetilde{F}_{0}(z)$ is the product of $F_{0}(z)$ (whose impulse response is $\left[\begin{array}{llll}1 & 1 & \ldots & 1\end{array}\right]$ ) and $W_{0}(z)$. Using (5), we can implement the transmitter efficiently. For each input vector, the transmitter only needs to carry out one $M$-point IDFT and implement one filter $W_{0}(z)$.

Comparison of Per Tone Shaping Filters and Time-domain Windowing Method: Per tone shaping filters have three main advantages over time-domain windowing method. To illustrate these advantages, we consider the VDSL downstream transmission and the PSD mask for the FTTCab M1 deployment scenario[3]. The system parameters are the same as those in Sec. 4. The PSD mask is shown in both Fig. 3(a) and (b). Firstly, in the per tone shaping filter technique, the window $W_{k}(z)$ can be chosen to be asymmetric. We are able to obtain bandpass filters $\widetilde{F}_{k}(z)$ that has a very sharp transition band on one side. Fig. 3(a) shows the filter response at $f=3.675 \mathrm{MHz}$. On the other hand, if the windows are frequency shifted version of a prototype $W_{0}(z)$, the prototype has to be designed so that all filters $\widetilde{F}_{k}(z)$ are good filters. Hence $W_{0}(z)$ is symmetric. Fig. 3(b) shows the corresponding filter response at $f=3.675 \mathrm{MHz}$. From the figure, one can clearly see the advantage of using an asymmetric shaping filter. Secondly, at different frequency regions, there are different requirements on the transition bandwidth and stopband attenuation of $\widetilde{F}_{k}(z)$. The per tone shaping filter technique allows us to design different windows for different requirements. For examples, for tones that are far from the shape edge of the PSD mask, one can choose a shaping filter with a larger transition bandwidth. There is no such flexibility in the time-domain windowing method. Thirdly, one can use shaping filters of different length for different $k$. A shorter filter causes less ISI when the order of channel $C(z)$ is larger than $L-P$. Though the per tone shaping filter technique has many advantages, its complexity is much higher than that of time-domain windowing method. A direct implementation of the transmitter in Fig. 2 has a complexity of $\mathcal{O}(M N)$. In what follows, we derive an efficient implementation of the transmitter with per tone shaping filters.

\section{EFFICIENT IMPLEMENTATION}

Per tone shaping filter technique has been proposed in [4] to improve the frequency responses of DMT receiving filters. It has been demonstrated that such a technique can greatly enhance the performance of DMT systems especially in the presence of narrowband noise. Moreover using the idea of sliding window fast Fourier transform, the authors have derived an efficient implementation of receivers with per tone shaping filters. In this section, we will show that using a procedure that is similar to that in [4], we are 
(a)

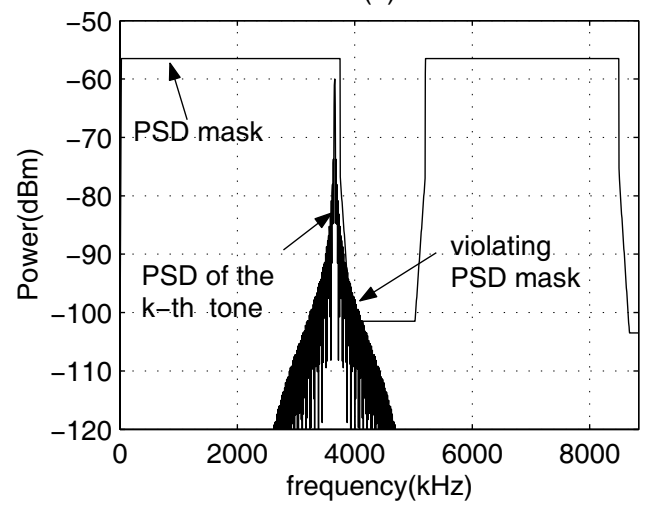

(b)

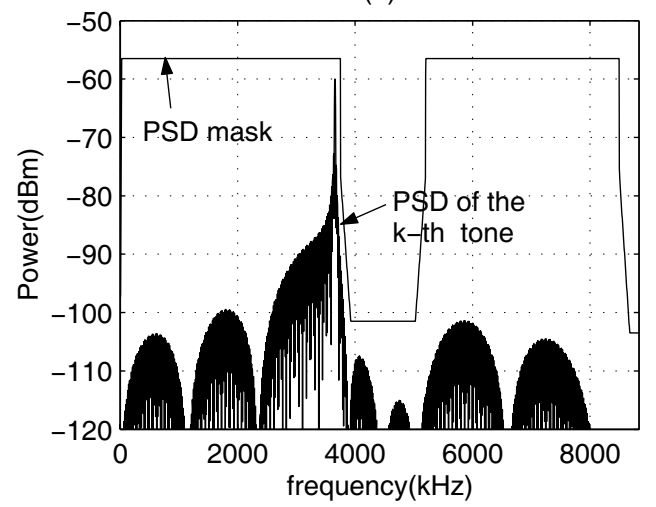

Fig. 3. Comparison of PSD for (a) the frequency shifted window and (b) the per tone window.

able to derive an efficient implementation of the DMT transmitter with per tone shaping filters.

Let the input vector $\mathbf{s}$ be

$$
\mathbf{s}=\left[\begin{array}{llll}
s_{0} & s_{1} & \ldots & s_{M-1}
\end{array}\right]^{T} .
$$

Consider the one shot response of the transmitter caused by the input vector $\mathbf{s}$. Let us denote one output block of the transmitter by

$$
\mathbf{x}=\left[\begin{array}{llll}
x(0) & x(1) & \ldots & x(N+P)
\end{array}\right]^{T} .
$$

Then from Fig. 2, we can write

$$
\mathbf{x}=\sum_{k=0}^{M-1} s_{k} \mathbf{f}_{\mathbf{k}} \star \mathbf{w}_{\mathbf{k}}
$$

where $\mathbf{f}_{\mathbf{k}} \star \mathbf{w}_{\mathbf{k}}$ denotes the convolution of $\mathbf{f}_{\mathbf{k}}$ and $\mathbf{w}_{\mathbf{k}}$. The vectors $\mathbf{f}_{\mathbf{k}}$ and $\mathbf{w}_{\mathbf{k}}$ are respectively given by

$$
\mathbf{f}_{\mathbf{k}}=\left(\begin{array}{c}
\alpha^{k L} \\
\alpha^{k(L-1)} \\
\vdots \\
\alpha^{k(L-N+1)}
\end{array}\right), \quad \mathbf{w}_{\mathbf{k}}=\left(\begin{array}{c}
w_{k}(0) \\
w_{k}(1) \\
\vdots \\
w_{k}(P)
\end{array}\right)
$$

Define the following $(N+P)$ by $(P+1)$ Toeplitz matrix

$$
\mathbf{T}_{k}=\left(\begin{array}{cccc}
\alpha^{k L} & 0 & \cdots & 0 \\
\alpha^{k(L-1)} & \alpha^{k L} & \ddots & \vdots \\
\vdots & \alpha^{k(L-1)} & \ddots & 0 \\
\alpha^{k(L-N+1)} & \vdots & \ddots & \alpha^{k L} \\
0 & \alpha^{k(L-N+1)} & \ddots & \alpha^{k(L-1)} \\
\vdots & \ddots & \ddots & \vdots \\
0 & \cdots & 0 & \alpha^{k(L-N+1)}
\end{array}\right) .
$$

Then one can express (6) as

$$
\mathbf{x}=\sum_{k=0}^{M-1} s_{k} \mathbf{T}_{k} \mathbf{w}_{\mathbf{k}}
$$

Let us decompose the matrix $\mathbf{T}_{k}$ as

$$
\mathbf{T}_{k}=\left(\begin{array}{cc}
\mathbf{f}_{\mathbf{k}} & -\alpha^{k(L+1)} \mathbf{I}_{P} \\
& \mathbf{0}_{(N-P) \times P} \\
\mathbf{0}_{P \times 1} & \alpha^{k} \mathbf{I}_{P}
\end{array}\right) \mathbf{U}_{k}
$$

where $\mathbf{U}_{k}$ is an $(P+1)$ by $(P+1)$ upper triangular Toeplitz matrix whose first row is given by $\left[\begin{array}{llll}1 & \alpha^{k} & \ldots & \alpha^{k P}\end{array}\right]$. Letting $\mathbf{v}_{k}=\mathbf{U}_{k} \mathbf{w}_{\mathbf{k}}$, we can rewrite (7) as

$$
\mathbf{x}=\sum_{k=0}^{M-1}\left(\begin{array}{cc}
\mathbf{f}_{\mathbf{k}} & -\alpha^{k(L+1)} \mathbf{I}_{P} \\
& \mathbf{0}_{(N-P) \times P} \\
\mathbf{0}_{P \times 1} & \alpha^{k} \mathbf{I}_{P}
\end{array}\right) \mathbf{v}_{k} s_{k} .
$$

Let us write $\mathbf{v}_{k}=\left[v_{k, 0} \overline{\mathbf{v}}_{k}^{T}\right]^{T}$. Thus $\overline{\mathbf{v}}_{k}$ is a $P$ by 1 vector consisting of the last $P$ entries of $\mathbf{v}_{k}$. Using this, one can verify that (8) can be written as

$\mathbf{x}=\left(\begin{array}{cc}\mathbf{0} & \mathbf{I}_{L} \\ \mathbf{I}_{M} \\ \mathbf{0}_{P \times M}\end{array}\right) \boldsymbol{\Phi}^{H} \mathbf{D} \mathbf{s}+\sum_{k=0}^{M-1}\left(\begin{array}{c}-\alpha^{k(L+1)} \overline{\mathbf{v}}_{k} \\ \mathbf{0}_{(N-P) \times 1} \\ \alpha^{k} \overline{\mathbf{v}}_{k}\end{array}\right) s_{k}$,

where $\mathbf{D}$ is the diagonal matrix $\operatorname{diag}\left[v_{0,0} v_{1,0} \ldots v_{M-1,1}\right]$ and $\boldsymbol{\Phi}^{H}$ is the IDFT matrix. Recall that (9) is the one shot response of the transmitter. When the input vectors $\mathbf{s}(n)$ are sent, there will be overlapping of $P$ samples between 2 adjacent blocks of $\mathbf{x}(n)$. Exploiting this overlapping section, one can verify that the computation of the second term in (9) needs roughly $P$ multiplications only. The cost of computing one transmitter output block is therefore roughly equal to the cost of $1 \mathrm{IDFT}$ and $(P+1) M$. In practice $P$ is often much smaller than $N$, the implementational cost of the efficient structure based on (9) is much lower than the direct implementation, which is $\mathcal{O}(M N)$. Comparing with the complexity of the frequency shifted window case, the proposed per tone window technique is higher by roughly $(P+1) M$ multiplications. This extra cost can be further reduced by chosen unequal length shaping filters. When the order of $W_{k}(z)$ is $P^{\prime}<P$, using $\mathbf{v}_{k}=\mathbf{U}_{k} \mathbf{w}_{\mathbf{k}}$, one can show that $\mathbf{v}$ has only $P^{\prime}+1$ nonzero entries. 
(a)

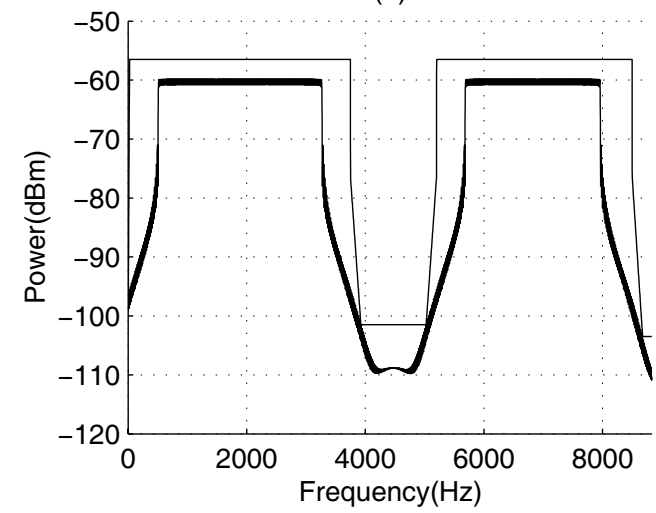

(b)

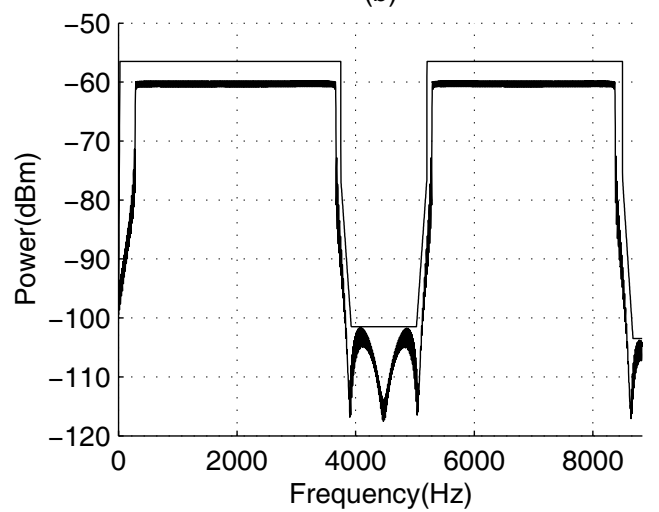

Fig. 4. PSD for transmitted signal $x(n)$ : (a) the frequency shifted window case; (b) the per tone window case.

\section{DESIGN EXAMPLE AND SIMULATION}

In the design example, we consider the VDSL downstream transmission and the PSD mask for the FTTCab M1 deployment scenario is used [3]. The PSD mask is shown in both Fig. 3. The system parameters are chosen as:

$$
\begin{aligned}
\operatorname{DFT} \operatorname{size}(M) & =1024 \\
\text { \# of redundant samples }(N-M) & =80 \\
\text { order of shapingfilters }(P) & =16 .
\end{aligned}
$$

The $k$ th shaping filter $W_{k}(z)$ is optimized so that the following cost function is minimized under the constraint (3).

$$
\begin{array}{r}
\min _{\mathbf{w}_{\mathbf{k}}}(1-\beta) \cdot \int_{\Omega_{1}}\left|F_{k}\left(e^{j \omega}\right) W_{k}\left(e^{j \omega}\right)\right|^{2} d \omega+ \\
\beta \cdot \int_{\Omega_{2}}\left|F_{k}\left(e^{j \omega}\right) W_{k}\left(e^{j \omega}\right)\right|^{2} d \omega,
\end{array}
$$

where $\Omega_{1}$ corresponds to the frequency bands not used for downstream transmission and this frequency range is

$$
\left[\begin{array}{ll}
0 & 4
\end{array}\right] \mathrm{kHz} \cup\left[\begin{array}{ll}
3.925 & 5.025
\end{array}\right] \mathrm{MHz} \cup\left[\begin{array}{ll}
8.675 & 30
\end{array}\right] \mathrm{MHz},
$$

and

$$
\Omega_{2}=\left[0, \frac{2 \pi(k-1)}{M}\right] \cup\left[\frac{2 \pi(k+1)}{M}, 2 \pi\right] .
$$

The constant $0 \leq \beta \leq 1$ is in general chosen to be close to 0 so that more weight is put on the stopband region of the PSD mask. In our example, we found $\beta=5 \times 10^{-4}$ to be a good choice. The reason for including the second term in the cost function is to prevent $W_{k}\left(e^{j \omega}\right)$ from amplifying the stopband of $F_{k}\left(e^{j \omega}\right)$ (which can result in a signficant increase of transmitting power). The optimal solution can be easily solved by using the least-squares method. Fig. 4(b) shows the PSD of transmitted signal $x(n)$ when a total of 375 tones (out of 512 tones) are allocated with equal transmission power. The average transmission power per tone is -90.3881 $\mathrm{dBm}$. Thus for DMT systems with per tone shaping filters, we are able to transmit data using 375 tones. For comparison, we have plotted the PSD of $x(n)$ for the time-domain windowing method for the same system parameters. The prototype window $W_{0}(z)$ is a Kaiser window with cutoff frequency $=0.01 \pi$. This cutoff frequency is chosen so that the best result is obtained. The resulting PSD when a total of 292 tones are transmitting data is shown in Fig. 4(a). The average transmission power per tone is -90.3813 $\mathrm{dBm}$. Note that because of the constraint in (3), the two systems have the same BER performance. But the per tone shaping filter technique allows us to utilize 375 tones for data transmission whereas the time-domain windowing method allows us to transmit data on 292 tones only. The gain is more than $28 \%$.

\section{CONCLUSIONS}

In this paper, we have proposed a DMT scheme with per tone transmitting shaping filters. An efficient implementation of the proposed system is derived. Simulation shows that for VDSL downstream transmission, the proposed per tone window technique has a much better spectral efficiency than the time-domain windowing method.

\section{REFERENCES}

[1] T. Starr, J. M. Cioffi and P. J. Silverman, Understanding DSL Technology, Prentice Hall, Inc., 1999.

[2] P. P. Vaidyanathan, Multirate systems and filter banks, Prentice Hall, Inc., 1993.

[3] "Interface Between Networks and Customer Installations Very-high Speed Digital Subscriber Lines(VDSL) Metallic Interface," American National Standards Institute, T1E1.4/2002031R2, 2002.

[4] K. Van Acker, G. Leus, M. Moonen, O. van de Wiel, and T. Pollet, "Per tone equalization for DMT-based systems," IEEE Trans. on Comm., pp. 109-119, Jan. 2001. 